\title{
Phase III Large Scale Vaccine Trial
}

National Cancer Institute

\section{Source}

National Cancer Institute. Phase III Large Scale Vaccine Trial. NCI Thesaurus. Code C15873.

Select suitable vaccine candidates and support efficacy trials when appropriate criteria are met. 